\title{
NECESIDAD DE REVISAR LAS INTERVENCIONES SANITARIAS PROMOVIDAS POR EL ESTADO PARA MUJERES EN PERÚ
}

\author{
Zoila Olga Romero-Albino (1) 1,a, Rafael Omar Domínguez-Samamés (101,b, \\ Maritza Ortiz-Arica (1) ${ }^{2, b}$, María Sofía Cuba-Fuentes (10 ${ }^{3, a}$ \\ 1 Universidad Peruana de Ciencias Aplicadas, Lima, Perú. \\ Ministerio de Salud, Lima, Perú. \\ 3 Universidad Peruana Cayetano Heredia, Lima, Perú. \\ Médico de familia y comunidad; ${ }^{\mathrm{b}}$ médico cirujano.
}

\section{RESUMEN}

Se describen las principales intervenciones sanitarias de promoción de salud y de prevención de enfermedades que se deben realizar en las mujeres en el sistema sanitario peruano. Se realizó una revisión de documentos técnicos normativos y de las recomendaciones de las principales organizaciones para prevención a nivel mundial. Dentro de las actividades de prevención se incluyeron la actividad física, alimentación saludable, consejería antitabaco e inmunizaciones. Además, se detallaron los principales tamizajes a realizar en las mujeres, tales como depresión, violencia, riesgo cardiovascular, citología de cuello uterino, mamografía, cáncer de colon; y dentro del espectro de prevención cuaternaria, se detallaron intervenciones que no han demostrado evidencia de beneficio para las mujeres. Las intervenciones sanitarias que se ofrecen desde el sistema sanitario peruano para las mujeres, al estar centradas meramente en aspectos reproductivos, pierden la concepción de integralidad que debe primar para el mantenimiento de la salud. En ese sentido, se propone el desarrollo de estrategias que no solo tengan evidencia, sino que sepan responder a las necesidades de las mujeres en el contexto peruano.

Palabras clave: Tamizaje Masivo; Estrategias Sanitarias; Salud de la Mujer (fuente: DeCS BIREME).

\section{NEED TO REVIEW SANITARY INTERVENTIONS PROMOTED BY THE GOVERNMENT FOR WOMEN IN PERU}

\begin{abstract}
The main health interventions for health promotion and disease prevention that should be performed in women in the Peruvian health system are described. A review of normative technical documents and the recommendations of the main organizations for worldwide prevention was carried out. The prevention activities included physical activity, healthy eating, tobacco counseling, immunizations; In addition, the main screening for women, such as depression, violence, cardiovascular risk, cervical cytology, mammography, colon cancer, are detailed; and within the spectrum of quaternary prevention, interventions that have not shown evidence of benefit to women are detailed. The health interventions that are offered from the Peruvian health system for women, being merely focused on reproductive aspects, lose the conception of integrality that should prevail for the maintenance of health. In that sense, it is proposed to develop strategies that not only have evidence, but also know how to respond to the needs of women in the Peruvian context.
\end{abstract}

Citar como: Romero-Albino ZO, Domínguez-Samamés RO, Ortiz Arica M, Cuba-Fuentes MS. Necesidad de revisar las intervenciones sanitarias promovidas por el estado para mujeres en Perú. Rev Peru Med Exp Salud Publica. 2020;37(1):129-35. Doi: https://doi. org/10.17843/rpmesp.2020.371.5097

Correspondencia: María Sofía Cuba Fuentes; Calle Jose Gonzales 775, dpto.604, Miraflores; maria.cuba@ upch.pe

Recibido: $13 / 01 / 2020$

Aprobado: 26/02/2020

En línea: 23/03/2020
Keywords: Mass screening; Health strategies; Women's health (source: MeSH NLM).

\section{INTRODUCCIÓN}

Los sistemas de salud tienen como uno de sus principales fines la mejora de los resultados sanitarios: disminuir la enfermedad y la muerte. En la consecución de este objetivo, los profesionales de salud diariamente realizan diagnósticos, hacen elecciones de exámenes y tratamientos y toman decisiones que impactan en la vida de las personas y sus familias ${ }^{(1,2)}$.

Las mujeres utilizan más los servicios sanitarios y suelen estar expuestas a intervenciones sanitarias de diversa índole ${ }^{(3)}$, que muchas veces están relacionadas a eventos fisiológicos que se empiezan a tratar como enfermedades; por ejemplo, el embarazo, la sexualidad y la menopausia, muchas veces abordados con intervenciones innecesarias. 
Algunos autores como Juan Gérvas y Mercedes Pérez-Fernández plantean que la desigual distribución de poder entre hombres y mujeres en sociedades patriarcales como las nuestras, también tiene consecuencias en la atención clínica ${ }^{(4)}$. Ser paciente mujer, dicen Gérvas y Pérez-Fernández, implica recibir peor atención que los hombres ya sea por defecto (acceso retrasado a programas de hemodiálisis, diagnóstico de insuficiencia respiratoria crónico) o por exceso (más apendicetomías innecesarias, mayor polimedicación que los hombres) ${ }^{(4)}$.

Una discriminación que se multiplica aún más cuando la mujer tiene una minusvalía, un problema mental grave, es anciana ${ }^{(5)}$, no es heterosexual ${ }^{(4,6,7)}$ o intercambia sexo por dinero o drogas ${ }^{(4)}$.

El concepto de normalidad muchas veces es un punto de corte arbitrario que se decide por estudios que indican desde cuándo puede haber más riesgo para la salud o por consenso de expertos. Algunos diagnósticos frecuentes como hipertensión arterial, diabetes mellitus u osteoporosis, se basan en curvas de normalidad. El ser catalogado como anormal puede ser el inicio de una serie de intervenciones que pueden tener un beneficio importante o pueden generar daño a hombres y mujeres ${ }^{(8)}$.

Las intervenciones sanitarias en las mujeres distan de como se visibilizan las intervenciones para la población masculina. En ese sentido, se alienta a virar hacia un sistema sanitario que propugne el desarrollo de intervenciones basadas en evidencia científica contextualizada a la realidad de la mujer peruana, logrando así mayor equidad; lo que repercutirá positivamente en la salud pública en general.

Este artículo busca describir las principales intervenciones sanitarias de promoción de salud y de prevención de enfermedad que se realizan en las mujeres en el sistema sanitario peruano a través de la normativa sanitaria nacional y sintetizar la mejor evidencia disponible de las intervenciones que son necesarias y señalar las que no tienen evidencia de efectividad.

\section{SESGOS DE LA DETECCIÓN TEMPRANA}

En general, debido a que la prevalencia de muchas enfermedades en una población asintomática suele ser baja, la mayoría de los resultados positivos de la detección serán falsos positivos (es decir, un valor predictivo positivo bajo). Los resultados falsos positivos, llevan a su vez a que los pacientes experimenten miedo, preocupación o ansiedad y generan una cascada diagnóstica terapéutica con costos al sistema sanitario o gasto de bolsillo de los pacientes y sus familias ${ }^{(9)}$.

Otros daños potenciales de la detección innecesaria incluyen resultados falsos negativos, que conducen a una falsa tranquilidad y sobrediagnóstico que ocurre cuando las personas son etiquetadas con amenaza de tener una en- fermedad que podría nunca ocasionarle daño y que puede llevar a la sobreutilización de exámenes y tratamientos. En ese sentido, cuando el tratamiento no es efectivo u ocasiona daños importantes, la detección temprana puede resultar en un daño en lugar de un beneficio ${ }^{(10)}$.

Los tamizajes se pueden definir como la aplicación de pruebas de diagnóstico en pacientes asintomáticos con el fin de dividirlos en dos grupos: aquellos que tienen una afección que se beneficiaría de una intervención temprana y aquellos que no lo hacen. El cribado se realiza para detectar la enfermedad en una etapa temprana, etapa asintomática cuando es potencialmente más susceptible al tratamiento. Sin embargo, adelantar el tiempo del diagnóstico solo no justifica la detección; el diagnóstico precoz también debe conducir a mejoras apreciables en la morbilidad y la mortalidad en comparación con la detección clínica de la enfermedad sintomática ${ }^{(11)}$.

\section{INTERVENCIONES DE PREVENCIÓN PRIMARIA CON EVIDENCIA DE BENEFICIO EN MUJERES}

Las mujeres deben ser aconsejadas para conseguir estilos de vida saludables que tendrán mayor impacto en su salud que muchas intervenciones desde el sistema de salud o que impliquen medicación. Sin embargo, los profesionales sanitarios debemos recordar que las condiciones en que vive la población tendrán impacto en su capacidad para desarrollar mejores estilos de vida.

A continuación, se describen intervenciones de prevención primaria para mujeres que han sido elegidas de acuerdo a la mejor evidencia y que pueden ser utilizadas en el ámbito de atención primaria.

Las recomendaciones relacionadas a estilos de vida saludables más importantes se describen a continuación y se resumen en la Tabla $1^{(12)}$ :

\section{a. Consejería antitabaco}

De acuerdo con la guía del Programa de Actividades Preventivas y de Promoción de la Salud (PAPPS), dejar de fumar implica superar la adicción a la nicotina, desaprender una conducta o modificar la influencia del medio en el que se desenvuelve el individuo; siendo recomendable realizar una intervención mediante la estrategia de las $5 \mathrm{~A}:^{(13)}$

Averiguar si el paciente fuma.

- Aconsejar a cada paciente que deje de consumir tabaco.

- Acordar objetivos y métodos apropiados que se basan en la intención del paciente a abandonar el tabaco.

- Ayudar al paciente en su intento de abandonar el tabaco, para lo cual se aconseja dejar de fumar, tratamientos farmacológicos disponibles u otros recursos, como llamadas 
Tabla 1. Estilos de vida saludables más importantes para las mujeres

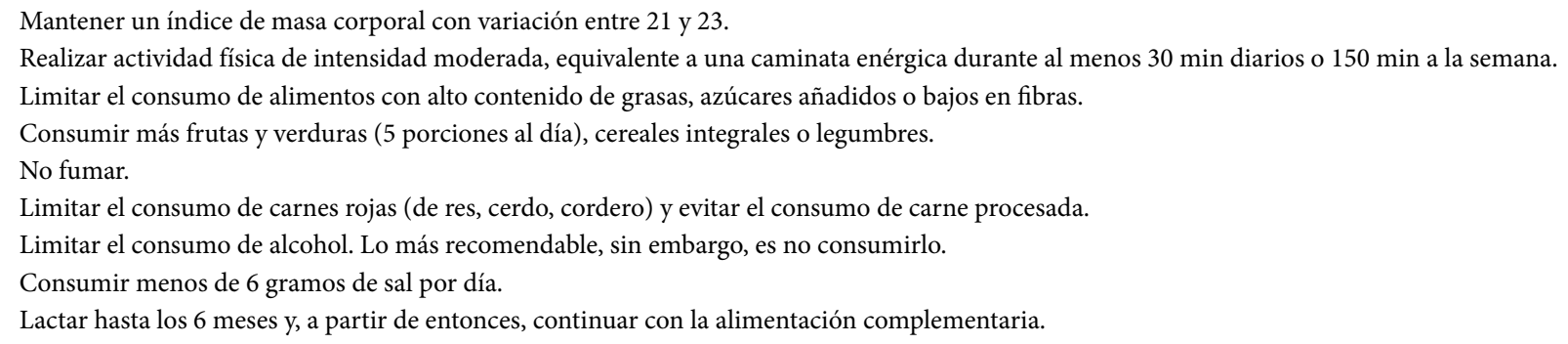

Adaptado de Recomendaciones de prevención del cáncer. Actualización PAPPS 2018

por teléfono o vía páginas web.

- Asegurarse vía internet a cada paciente, con el desarrollo de un plan de contingencia si en caso surge la recaída, fortalecimiento de redes de apoyo y nuevas rutinas.

\section{b. Vacunas}

Además de cumplir el calendario de vacunación durante la infancia. Existen algunas vacunas que pueden recomendarse en mujeres:

\section{Vacunas contra el virus del papiloma humano}

La infección del cuello uterino por el virus del papiloma humano es una enfermedad de transmisión sexual, donde en algunos casos la infección de las células epiteliales puede provocar cáncer a través de un proceso que puede durar varios años. Existen más de 200 tipos de virus del papiloma humano, pero cuatro son los de mayor poder oncogénico: $16,18,31$ y 45 ; la vacuna protege contra los virus $6,11,16$ y 18 y existe otra vacuna que protege contra los tipos 31,33 , 45,52 y 58 . Sin embargo, de acuerdo a los estudios, la vacuna es eficaz medianamente en mujeres entre los 16 y 45 que no han tenido relaciones sexuales y en lesiones precancerosas provocadas por los virus contra los que se les vacuna ${ }^{(14)}$.

La edad ideal para vacunarse contra el virus de papiloma humano es a partir de los 9 años, antes del inicio de la actividad sexual, pues así la vacuna confiere mejor protección. En el caso del calendario de inmunizaciones peruano, se establece para niñas y adolescentes que estén cursando el quinto grado de primaria regular, y para niñas de 9 años hasta los 13 años 11 meses 29 días que no se encuentren estudiando.

\section{Vacuna antitetánica}

La indicación de la vacunación es universal, en cinco dosis. En la mujer adulta con vacunación infantil completa se debe administrar una dosis de recuerdo a los 10 años desde la última dosis ${ }^{(15)}$. En Perú, se administra una mezcla de toxoide tetánico y diftérico a los adultos que recibieron el esquema completo contra estas enfermedades, cada 10 años para reforzar la inmunidad.

\section{Vacuna contra la influenza}

Se recomienda la vacunación de mujeres adultas mayores; las revisiones sistemáticas concluyeron que aquellos que recibieron la vacuna podrían tener menos riesgo para contraer la enfermedad (de $6 \%$ a $2,4 \%$ ) y probablemente menos riesgo de presentar síntomas (de $6 \%$ a 3,5\%) respecto a quienes no recibieron la vacuna ${ }^{\left({ }^{16)}\right.}$.

En el calendario de inmunizaciones peruano, esta vacuna se administra en niños menores de un año, y a partir de los tres a 59 años, siendo prioritaria la vacunación a aquellas personas que presentan alguna condición de comorbilidad, gestantes a partir de las 20 semanas y personas adultas mayores, de 60 años a más. Así mismo, establece algunos grupos de riesgo, tales como comunidades nativas, estudiantes de ciencias de la salud, fuerzas armadas, población de distritos en riesgo por bajas temperaturas, personal de la policía, Cruz Roja, Defensa Civil, bomberos, población privada de su libertad y personas que se encuentren en centros de rehabilitación u otros.

\section{Vacuna antineumocócica}

La vacuna antineumocócica previene infecciones invasivas graves causadas por neumococo, como neumonía, meningitis, septicemia. También previene formas no invasivas como otitis media aguda. La vacuna se debe administrar en tres dosis en niños y actúa para los serotipos 1, 3, 4, 5, 6A, 6B, 7F, 9V, 14, 18C, 19A, 19F y 23F. Los adultos mayores de 60 años o más deben recibir una dosis ${ }^{(17)}$. En el calendario de inmunizaciones peruano, esta vacuna se administra en niñas menores de 1 año, en las niñas de 2 a 4 años que presentan alguna comorbilidad y personas adultas mayores, de 60 años a más.

\section{PREVENCIÓN SECUNDARIA}

A continuación, se describen intervenciones de prevención secundaria, las cuales han sido elegidas de acuerdo a los problemas más prevalentes en las mujeres, así como a la mejor evidencia. Para el caso del marco normativo peruano, dichas intervenciones requieren mayor detalle en su desarrollo. 


\section{a. Tamizaje de depresión}

La detección precoz y tratamiento psicosocial mejoran la calidad de vida y pronóstico de los pacientes afectados por depresión. La entrevista clínica es el elemento más importante para la detección y tratamiento, que requiere de habilidades comunicativas por parte del entrevistador.

La detección de depresión en mujeres se debe realizar a partir de los 18 años e incluir a las personas adultas mayores, mujeres embarazadas y en posparto. Resultan útiles los instrumentos, como el Cuestionario de salud del paciente (PHQ, por sus siglas en inglés) en sus diversas formas, las Escalas de ansiedad y depresión hospitalarias en adultos, la Escala de depresión geriátrica en adultos mayores y la Escala de depresión posnatal de Edimburgo (EPDS, por sus siglas en inglés) en mujeres en posparto y gestantes. Cabe señalar que si se genera un resultado positivo en alguna de estas evaluaciones, es necesario realizar una evaluación adicional ${ }^{(18)}$.

\section{b. Tamizaje de violencia}

Existen diversos instrumentos para tamizar violencia en mujeres, es importante que los profesionales de salud estén entrenados para realizar una entrevista, aseguren la privacidad y confidencialidad, escuchen activamente y con actitud empática, observen la postura y el estado emocional, y eviten emitir juicios.

Dentro del perfil de la mujer susceptible a maltrato se identifica la dependencia, violencia intrafamiliar en familia de origen, bajo nivel económico y sociocultural, aislamiento social y psicológico, discapacidad, mujeres sumisas o con estereotipos ya arraigados, embarazo, consumo de alcohol y drogas, entre otros. En el caso de las mujeres adultas mayores, la vulnerabilidad está relacionada con la edad, incontinencia, deterioro cognitivo, aislamiento social, entre otros. Es importante hacer preguntas sobre violencia en estos grupos vulnerables.

De acuerdo al Grupo de trabajo sobre servicios preventivos de los Estados Unidos (USPSTF, por sus siglas en inglés), los instrumentos que pueden detectar violencia de pareja en el último año son HARK, que evalúa la violencia emocional y física en el último año, expresada como humillación, miedo, violación, patadas; HITS, que evalúa la frecuencia de la violencia; E-HITS, que evalúa a través de una pregunta adicional sobre violencia sexual a las anteriores; PVS, que incluye 3 elementos que evalúan el abuso físico y la seguridad; y WAST, herramienta de detección de abuso de mujeres, que incluye ocho preguntas que evalúan violencia física y emocional ${ }^{(19)}$.

\section{c. Tamizajes para prevenir problemas de salud cardiovasculares y metabólicos}

Es necesario que estas condiciones sean identificadas oportunamente en las mujeres, ya que muchas veces las mujeres reciben menos cuidados en la detección y manejo de estos problemas.
Hipertensión arterial: En mujeres adultas de 18 a 39 años con presión arterial (PA) en valores normales y sin factores de riesgo, evaluar la PA cada 3 a 5 años. En mujeres adultas de 40 años o más, o con factores de riesgo, evaluar anualmente ${ }^{(20)}$. De obtener un valor de PA superior a 140/90 mmHg, confirmar realizando monitorización ambulatoria de la presión arterial (MAPA), para evitar la instauración de tratamiento ante fenómenos como la bata blanca.

Diabetes mellitus: Según medición de la glicemia basal en ayunas ( $\geq 126 \mathrm{mg} / \mathrm{dL}$ ) o a las 2 horas tras sobrecarga oral con glucosa ( $\geq 200 \mathrm{mg} / \mathrm{dL}$ ) o según la medición de la hemoglobina glicosilada $(\geq 6,5 \%){ }^{(27)}$. Este tamizaje aplica sobre población adulta de entre 40 y 70 años, con sobrepeso u obesidad, sin síntomas de diabetes mellitus. Este tamizaje se repetiría cada tres años siempre que los resultados previos sean normales ${ }^{(21)}$.

Dislipidemia: Según medición del colesterol total $(\geq 200$ $\mathrm{mg} / \mathrm{dL})$ y del HDLc $(<45 \mathrm{mg} / \mathrm{dL})$. Este tamizaje aplicaría sobre población de 18 años o más, cada cuatro años. (Grupo de expertos del PAPPS) ${ }^{(22)}$.

\section{d. Citología de cuello uterino}

La citología es una prueba de cribado para diagnosticar cáncer de cuello uterino en fases precoces. Cuando los resultados presentan anormalidad, se calcula que las neoplasias intraepiteliales cervicales NIC 1 o NIC 2, desaparecen entre el 100\% y $40 \%$ de los casos; no así con las NIC 3 y el carcinoma in situ que pueden progresar a lo largo de los años hasta que generar cáncer.

Se ha encontrado, al realizar el seguimiento a mujeres entre 30 y 65 años con tres o más citologías consecutivas normales, que ninguna desarrollará cáncer de cuello uterino en el siguiente año: el valor predictivo positivo de la cuarta citología es del $1 \%$, por lo que, a partir del tercer Papanicolaou negativo, se recomienda espaciar su toma a una frecuencia de cada tres años para evitar el riesgo de falsos positivos y sobrediagnóstico.

El Papanicolaou debe ser recomendado cada tres años en mujeres entre los 21 y 29 años, y para las mujeres entre 30 y 65 años, detección por citología cada tres o cada cinco años, con pruebas de alto riesgo (hrHPV) solas o en conjunto con citología $^{(23)}$.

\section{e. Mamografía para cáncer de mama}

La mamografía no se constituye como una técnica diagnóstica, sino más bien como un filtro para el diagnóstico de cáncer de mama. El cribado por 10 años de 1000 mujeres entre 50 y 69 años, evita una muerte por cáncer de mama; sin embargo, diez mujeres serán tratadas sin que tuvieran cáncer (sobrediagnóstico) y se habrán producido falsos positivos en doscientas mujeres. En sentido absoluto se disminuye la mortalidad por cáncer de mama en un $0,1 \%$ y en sentido relativo disminuye un $21 \%$ la mortalidad ${ }^{(24)}$. Se recomienda que el cribado para cáncer de mama sea discutido con las pacientes y recomendado conociendo beneficios y riesgos de este en mujeres entre 50 y 65 años, cada dos años. 


\section{f. Tamizaje de cáncer de colon}

Las mujeres de 50 años a más presentan un riesgo medio de desarrollar cáncer colorrectal. Los factores de riesgo son la edad, la obesidad, el sedentarismo, la dieta rica en carne procesada y roja, el tabaquismo y alcoholismo, la poliposis colónica, la colitis ulcerosa y ser portador de mutaciones del síndrome de Lynch o síndrome de poliposis familiar.

Para el diagnóstico precoz, las pruebas de cribado con mayor evidencia son la sangre oculta en heces ( $\mathrm{SOH})$ o inmunológica (SOHi), con periodicidad bienal, la sigmoidoscopía (SG) cada cinco años, o la colonoscopia cada 10 años ${ }^{(25)}$.

\section{g. Densitometría}

La osteoporosis se constituye en uno de los factores de riesgo para fracturas; no obstante, son las caídas el principal factor de riesgo de fracturas, siendo la fractura de cadera la más grave en las personas adultas mayores.

La densitometría tiene un valor predictivo positivo (VPP) a los 70 años del 9\%; es decir, que, si una paciente de 70 años se somete a la prueba y se le diagnostica de osteoporosis, la probabilidad de que tenga en realidad la enfermedad es del 9\%. En el caso de las mujeres de 80 años, el VPP es del 36\%, y para mujeres de 60 años, los datos no son concluyentes; sin embargo, de acuerdo a algunos estudios se calcula en 5\%. Se recomienda, por lo tanto, solicitar densitometría como tamizaje solo en mujeres mayores de 65 años o que tengan un riesgo incrementado para el desarrollo este problema. ${ }^{(26)}$

\section{INTERVENCIONES SIN EVIDENCIA DE BENEFICIO EN MUJERES}

A continuación, nos referimos a las intervenciones que no demuestran evidencia de beneficios para las mujeres y que, sin embargo, son recomendadas muy frecuentemente por personal sanitario e incluidas en algunas normas técnicas del sector salud.

\section{a. Autoexploración mamaria}

La autoexploración, técnica que permite la revisión sistemática del tejido mamario, es recomendada en las normas técnicas sanitarias del Ministerio de Salud del Perú. Sin embargo, al realizar el seguimiento por 10 años de un grupo de mujeres que realizaron autoexploración, se duplicó el número de diagnósticos de lesiones benignas y el de biopsias; no existiendo ninguna diferencia en la mortalidad por cáncer de mama, con el subsecuente estrés al que conllevó el diagnóstico ${ }^{(27)}$. Por tanto, no se debería recomendar su realización.

\section{b. Cribado de cáncer de ovario}

La determinación rutinaria del marcador tumoral CA-125, la exploración ginecológica y la ecografía transvaginal para de- tectar cáncer de ovario no reducen la mortalidad por cáncer de ovario, y la evaluación diagnóstica después de un resultado falso positivo se asoció a complicaciones ${ }^{(28)}$. El diagnóstico precoz de cáncer de ovario no modifica la mortalidad, sino que conlleva a más sobrediagnóstico, intervenciones innecesarias y más efectos adversos.

\section{c. Terapia de reemplazo hormonal en la meno- pausia}

Es muy frecuente que los síntomas como sofocación, insomnio, sequedad vaginal se intenten medicalizar a través de la terapia de reemplazo hormonal. Sin embargo, de acuerdo a los estudios, se ha demostrado que esta puede provocar el desarrollo de tromboembolias, cáncer de mama e infartos. Se reconoce su utilidad por, ejemplo, en caso de mujeres jóvenes a las que se le ha extirpado los ovarios; no obstante, no es el caso de todas las mujeres. No se recomienda el uso de estrógenos y progestágenos combinados en mujeres posmenopáusicas de forma rutinaria ${ }^{(29)}$.

\section{d. Examen pélvico bimanual}

Aunque es indicado de forma rutinaria por profesionales de salud, en las mujeres asintomáticas no existe evidencia que avale el hacerlo de forma rutinaria o con periodicidad. Estudios poblacionales en mujeres jóvenes encontraron que el $54 \%$ son innecesarios. ${ }^{(30)}$.

No se identificaron pruebas directas de los beneficios y daños generales del examen pélvico como una prueba de detección periódica o por única vez. Se identificaron pruebas limitadas con respecto a la precisión diagnóstica y los daños de los exámenes pélvicos de detección de rutina en poblaciones asintomáticas de atención primaria.

En la Tabla 2 se presenta un resumen de recomendaciones para hacer y no hacer de intervenciones sanitarias en mujeres.

\section{CONCLUSIONES}

Es necesario considerar que la salud de la mujer en cualquiera de las etapas en la que se encuentre implica un reto para los profesionales de la salud y el sistema sanitario; en ese sentido, es necesario contar con profesionales, con competencias clínicas y de comunicación que les permita el acercamiento a las personas, el entendimiento de su contexto para la toma de decisiones compartidas.

Las intervenciones sanitarias deben poseer la mayor evidencia posible para su consideración, no estar basadas en la opinión de expertos exclusivamente e intentar estar desligadas de intereses de la industria farmacéutica, política, mercantilista o de otra índole.

Las intervenciones no basadas en evidencia en las mujeres pueden generar una cascada de intervenciones diagnósticas y terapéuticas que pueden generar preocupación en los 
Tabla 2. Intervenciones sanitarias en mujeres y observaciones relacionadas.

\begin{tabular}{|c|c|c|}
\hline Actividad & ¿Hacer? & Observaciones \\
\hline Recomendación para Actividad física & Sí & \\
\hline Recomendación en Alimentación saludable & Sí & \\
\hline Consejería antitabaco & Sí & \\
\hline Vacuna contra virus del papiloma humano & Sí & A partir de los nueve años, antes de iniciar actividad sexual. \\
\hline Vacuna antitetánica & Sí & En mujeres con vacunación infantil, cada 10 años. \\
\hline Vacuna contra la influenza & Sí & Mujeres adultas mayores. \\
\hline Vacuna antineumocócica & Sí & Niñas y adultas mayores. \\
\hline Tamizaje de depresión & Sí & PHQ, EPDS. \\
\hline Tamizaje de violencia & Sí & HARK, HITS, E-HITS, PVS, WAST. \\
\hline $\begin{array}{l}\text { Tamizaje para prevenir problemas de salud } \\
\text { cardiovasculares y metabólicos }\end{array}$ & Sí & $\begin{array}{l}\text { HTA: evaluar PA mujeres sin FR de } 18 \text { a } 39 \text { años, cada tres a cinco años. En mujeres } \\
\text { mayores de } 40 \text { años a más, con FR, anualmente. } \\
\text { DM: mujeres entre } 40 \text { y } 70 \text { años con sobrepeso u obesidad, sin síntomas de DM; cada } \\
\text { tres años con exámenes previos normales. } \\
\text { Dislipidemia: en mujeres mayores de } 18 \text { años cada cuatro año. }\end{array}$ \\
\hline Citología de cuello uterino & Sí & $\begin{array}{l}\text { En mujeres de } 21 \text { a } 29 \text { años cada tres años y en mujeres entre } 30 \text { y } 65 \text { años cada tres o } \\
\text { cinco años. }\end{array}$ \\
\hline Mamografía & Sí & Mujeres de 50 a 65 años cada dos años. \\
\hline Tamizaje de cáncer de colon & Sí & Mujeres de 50 a 74 años sin riesgo alto. \\
\hline Densitometría & Sí & Mujeres mayores de 65 años con riesgo incrementado. \\
\hline Autoexploración de mama & No & \\
\hline Cribado de cáncer de ovario & No & Considerar contexto \\
\hline $\begin{array}{l}\text { Terapia de reemplazo hormonal en la me- } \\
\text { nopausia }\end{array}$ & No & Considerar contexto \\
\hline Examen pélvico de rutina en mujeres & No & Considerar contexto \\
\hline
\end{tabular}

pacientes y gastos innecesarios para estas, sus familias o el sistema sanitario

Los profesionales de la salud en cualquier ámbito donde laboren deben propiciar espacios de discusión, reflexión y debate sobre las políticas sanitarias vigentes en el país y evitar el sobrediagnóstico y sobretratamiento, y buscar que las intervenciones con evidencia lleguen a la mayor parte de personas.

Para rediseñar el tipo de cuidado que se ofrece desde el sistema sanitario a las mujeres, debemos reflexionar en la forma en que este se realiza. Es posible que sigan prevaleciendo parámetros centrados en aspectos reproductivos sin tener en cuenta la diversidad de situaciones, contextos, sexualidades y estilos de vida de las mujeres y sus diferentes necesidades de salud.

Asimismo, es necesario considerar que desde cualquier nivel donde el profesional se desenvuelva debe ser crítico de la información, de tal manera que canalice a instancias de la gestión que corresponda, propuestas de intervenciones con evidencia, siempre en aras de la construcción de un mejor sistema de salud. Los programas presupuestados pueden ser una alternativa para esta canalización, a fin de que estas propuestas obtengan el financimiento que corresponde. Otra de las vías para canalizar estas propuestas es a través de la sociedad civil, logrando consensuar diversas alternativas donde no solo prime el carácter científico, sino los diferentes contextos del ámbito nacional.

Contribución de los autores: ZORA, RODS, MOA y MSCF han participado en la concepción del artículo, su redacción y aprobación de la versión final.

Fuentes de financiamiento: Autofinanciado.

Conflictos de interés: Los autores declaran no tener conflictos de interés.

\section{REFERENCIAS BIBLIOGRÁFICAS}

1. Sackett D. The arrogance of preventive medicine. CMAJ.2002;167(4):363-364.

2. Coll-Benejam T, Bravo-Toledo R, Marcos-Calvo M, Astier Peña M. Impacto del sobrediagnóstico y sobretratamiento en el paciente, el sistema sanitario y la sociedad. Aten Primaria. 2018;50(S2):86-95. doi: 10.1016/j. aprim.2018.08.004
3. Gomez E. Género, equidad y acceso a los servicios de salud: una aproximación empírica. Rev Panam Salud Publica. 2002;11(5/6):327-334.

4. Gérvas J, Pérez-Fernández M. El encarnizamiento médico con las mujeres: 50 intervenciones sanitarias excesivas y cómo evitarlas. Primera edición. Barcelona: Editorial Los Libros del Lince; 2016. 
5. Guarnizo-Herreño C, Agudelo C. Equidad de Género en el Acceso a los servicios de salud en Colombia. Rev Salud Pública. 2008; 10(1):44-57.

6. Marshal MP, Friedman MS, Stall R, King KM, Miles J, Gold MA, Bukstein OG, Morse JQ. Sexual orientation and adolescent substance use: a meta-analysis and methodological review. Addiction. 2008;103(4):546-556.

7. Reisner SL, Poteat T, Keatley J, Cabral M, Mothopeng T, Dunham E. Global health burden and needs of transgender populations: a review. The Lancet. 2016;388:412-436. doi: 10.1016/S0140-6736(16)00684-X.

8. Rose G. Sick individuals and sick populations. Int J Epidemiol. 2001;30(3):427-32.

9. McWhinney I. Illnes in the Community. In: McWhinney I. Textbook of family medicine. New York: Oxford University Press Inc.; 2009:30-8.

10. Gates TJ. Screening for Cancer: Concepts and Controversies. Am Fam Physician.2014;90(9):625-631.

11. Gérvas. J, Pérez-Fernández M. Sano y salvo y libre de intervenciones sanitarias innecesarias. Barcelona: Los libros del Lince; 2013.

12. Marzo-Castillejo M, Vela-Vallespín C, Bellas-Beceiro B, Bartolomé-Moreno C, Melús-Palazón E, Vilarrubí-Estrella M, Nuin-Villanueva M. Recomendaciones de prevención del cáncer. Actualización PAPPS 2018. Aten Primaria. 2018;50:41-65. doi: 10.1016/S0212-6567(18)30362-7

13. Córdoba R, Camarelles F, Muñoz E, Gómez J, SanJosé J, Ramírez J, Martín C, Campo M, Revenga J. Recomendaciones sobre el estilo de vida. Actualizacón PAPPS 2018. Aten Primaria. 2018;50:29-40. doi: 10.1016/S0212-6567(18)30361-5

14. Gavilán E, Padilla J.La vacuna del papiloma humano. AMF.2013;9(4):201-207.

15. Grupos de expertos del PAPPS. Prevención de las enfermedades infecciosas. Actualización PAPPS en vacunas 2018. Aten Primaria. 2018;50(Supl 1):66-82. doi: 10.1016/S0212-6567(18)30363-9

16. Demicheli V, Jefferson T, Di Pietrantonj C, Ferroni E, Thorning S, Thomas RE, Rivetti A. Vaccines for preventing influenza in the elderly. Cochrane Database Syst Rev. 2010 Feb 17;(2):CD004876. doi: 10.1002/14651858. CD004876.pub3.

17. Siu AL, and the US Preventive Services Task Force (USPSTF). Screening for depression in adults: us preventive services task force recommendation statement. JAMA. 2016;315(4):380-387. doi:https://doi.org/10.1001/ jama.2015.18392.

18. Buitrago F, Ciurana R, Chocrón L, Fernández M, García J, Montón C, Tizón J. Prevención de los trastornos de la salud mental en atención primaria. Actualización PAPPS 2018. Aten Primaria. 2018;50:83-108. doi: 10.1016/S0212-6567(18)30364-0

19. US Preventive Services Task Force. Screening for Intimate Partner Violence, Elder Abuse, and Abuse of Vulnerable Adults: US Preventive Services Task Force Final Recommendation Statement. JAMA. 2018;320(16):1678-1687. doi:https://doi.org/10.1001/jama.2018.14741.
20. Final Recommendation Statement: High Blood Pressure in Adults: Screening. U.S. Preventive Services Task Force. April 2019. https://www. uspreventiveservicestaskforce.org/Page/Document/RecommendationStatementFinal/high-blood-pressure-in-adults-screening.

21. Final Recommendation Statement: Abnormal Blood Glucose and Type 2 Diabetes Mellitus: Screening. U.S. Preventive Services Task Force. April 2018. https://www.uspreventiveservicestaskforce.org/Page/Document/ RecommendationStatementFinal/screening-for-abnormal-blood-glucose-and-type-2-diabetes.

22. Brotons Cuixart C, Alemán Sánchez JJ, Banegas Banegas JR, Fondón León C, Lobos-Bejarano JM, Martín Rioboó E, et al. Recomendaciones preventivas cardiovasculares. Actualización PAPPS 2018. Aten Primaria. 2018;50(Supl 1):4-28. doi: 10.1016/S0212-6567(18)30360-3.

23. Curry SJ, Krist AH, Owens DK, Barry MJ, Caughey AB, Davidson KW, et al. Screening for Cervical Cancer US Preventive Services Task Force Recommendation Statement. JAMA. 2018;320(7):674-686. doi:10.1001/ jama.2018.10897.

24. Cochrane Breast Cancer Group. The Cochrane Library Enero 2011. [Internet] Disponible en: http://onlinelibrary.wiley.com/doi/10.1002/14651858. CD001877.pub4/abstract;jsessionid=2F9C8235C1DE434D71B82ADB F6F2E44C.d03t04.

25. Gupta N, Kupfer SS, Davis AM. Colorectal Cancer Screening. JAMA. 2019;321(20):2022-2023. doi:https://doi.org/10.1001/ jama.2019.4842

26. Wilkin TJ, Devendra D. Bone densitometry is not a good predictor of hip fracture. BMJ.2001; 323:795. doi: https://doi.org/10.1136/ bmi.323.7316.795.

27. Kösters JP, Gøtzsche PC. Regular self-examination or clinical examination for early detection of breast cancer. Cochrane Database Syst Rev. 2003;2:CD003373. DOI: 10.1002/14651858.CD003373.

28. Buys S, Partridge E, Black A, Johnson CC, Lamerato L, Isaacs C, et al. Effect of screening on ovarian cancer mortality. The Prostate, Lung, Colorectal and Ovarian (PLCO) Cancer Screening Randomized Controlled Trial. JAMA. 2011;305(22):2295-2303. doi:10.1001/jama.2011.766.

29. Moyer V, U.S. Preventive Services Task Force. Menopausal Hormone Therapy for the Primary Prevention of Chronic Conditions: U.S. Preventive Services Task Force Recommendation Statement. Ann Intern Med. 2013;158(1):47-54.

30. Quin J, Saraiya M, Martinez G, Sawaya GF. Prevalence potencially unnecessary bimanual pelvic examinations and Papanicolaou tests among adolescent girls and young women aged 15-20 years in the United States. JAMA Intern Med. 2020 Jan 6. doi: 10.1001/jamainternmed.2019.5727. 